\title{
LAS REVISTAS LITERARIAS EN CASTILLA Y LEÓN EN LOS AÑOS 20. UN FENÓMENO A LA CABEZA DE LA VANGUARDIA
}

\author{
Mercedes Andrés López
}

\begin{abstract}
Literary journals are at the peak in $20^{\text {th }}$ century in Spain. Some examples are "Cruz y Raya" or "Hora de España". They were also used by authors as a showcase to show their productions. In Castile and Leon, in the 1920s three publications emerged: "La Cotorra" (Soria), "Manatial" (Segovia) and "Parábola" (Burgos). All of them will be an example of avant-garde. The first one because of its jocular character, which departs from the expected. The second one because of the ultraism of its poetry. And the incorporation of images. The last one shows a plain ultraism.
\end{abstract}

Keywords: Literary journals; avant-garde; Castile and Leon; modernization; ultraism.

Resumen: Las revistas literarias personifican un fenómeno en pleno auge en el siglo XX español, dando conocidos frutos como "Cruz y Raya" u "Hora de España" y siendo utilizadas por los autores que formaban parte de sus equipos de redacción como escaparate en el que dar a conocer su obra. En los años 20, en la región de Castilla y León surgen tres publicaciones diganas de mención: “La Cotorra (Soria), “Manatial" (Segovia) y "Parábola" (Burgos), que serán un ejemplo de vanguardia, entendida de diversas formas. La primera por su carácter jocoso, rompiendo con lo esperado; la segunda por su carácter ultraísta en lo poético y por la incorporación de la imagen, y la tercera dando muestras de ultraísmo más puro.

Palabras claves: Revistas literarias; vanguardia; Castilla y León; renovación; ultraísmo.

\section{La revista literaria como fenómeno literario de vanguardia en Castilla y León}

El fenómeno de las revistas literarias resulta sin duda singular, especialmente si tenemos en cuenta que el gran auge - sobre todo en España - de este movimiento se da exclusivamente en el siglo XX. Ya se dieron ciertos ejemplos de revistas literarias en Europa a lo largo de los siglos XVIII y XIX, si bien escasos y no definidos dentro de un todo como ocurrirá en el siglo venidero.

La revista literaria, por tanto, adquiere una autonomía y una personalidad propia en el siglo XX, en el que podemos decir que encontramos ya un esqueleto característico de este tipo de publicaciones que comparten rasgos e identidad común.

Castilla y León ha sido bien conocida a lo largo de la historia de la literatura como una región de secas y áridas letras, de escasa innovación y ruptura, de falta de vanguardia. La imagen de los Campos de Castilla machadianos, del hombre castellano tradicional, duro 
y ajeno a nuevos movimientos han dejado mella en la idea que se tiene de nuestra literatura regional, tanto dentro como fuera de sus fronteras.

Sin embargo, poco de cierto hay en esto si tenemos en cuenta que la zona fue fuente de diversos movimientos artísticos y literarios que fueron vanguardia y que hoy estudiamos, muchas veces sin siquiera pensar en que sean castellanos, como vanguardistas.

Refiriéndonos concretamente a los años 20 no podemos dejar de pensar en la generación del 27, siempre relacionada con Madrid, con la Residencia de Estudiantes, con las tertulias literarias, y por qué no, con la Granada que ha sabido sacar jugo de Lorca y la Orihuela que en el último año más que nunca, nos ha vendido a Miguel Hernández como producto literario e incluso turístico y comercial. Permitámonos aquí realizar una reflexión social, e incluso política, ¿no es cierto que en muchas ocasiones hemos dejado de vender la calidad literaria de lo acontecido en Castilla? ¿Qué hemos dejado pasar la oportunidad de darnos a conocer como una tierra de vanguardia en lo literario? ¿Qué muchos de nuestros autores más conocidos no se relacionan jamás con su lugar de origen o aquellos espacios que marcaron en parte su producción literaria? Lejos de querer con esto dar lugar a una reflexión chovinista sobre lo que existe en Castilla y lo que esta región ha aportado a la literatura lo que pretendo es dar a conocer una realidad que por motivos políticos, de mala gestión del patrimonio cultural, o hablando en un modo más coloquial, de mala visión comercial y turística ha pasado por la historia de la literatura entrevista por un velo de luces y sombras.

La vanguardia en Castilla y León está en las glosas silenses, en la imprenta Román y Fadrique que vio florecer La Celestina en papel, en el Machado más soriano, en el Poema de Mio Cid, en María Zambrano, en la Zamora de León Felipe, en los versos de la muerte de Jorge Manrique. Sí, ahí está. Pero, ¿qué hay de un fenómeno tan importante como el de las revistas literarias, prácticamente olvidado por los manuales de literatura? Estas publicaciones eran la vanguardia llevada a la calle, el papel nuestro de cada día de los ciudadanos de a pie, la literatura de la calle y del hombre intelectual de la época. Y es innegable que estas revistas tuvieron un especial florecer en Castilla y León. Ejemplos como Parábola, Claraboya, El cobaya, La cotorra, Artesa, Ateneo o Manantial, entre muchas otras, lo demuestran.

Por tanto son dos los motivos principales para pararse a reflexionar sobre el asunto: el primero de ellos reside en el afán de defender la faceta de vanguardia que ha acompañado siempre a la región de Castilla y León a lo largo de la historia de la literatura, y que por motivos diversos ha pasado desapercibido - o al menos no ha sido tan perceptible como en mi opinión se merece - a los ojos de la crítica y el lector habitual. El segundo consiste en rescatar, dentro de esta idea de la vanguardia en la región, el fenómeno de la revista literaria, un punto clave en la innovación y que en muchas ocasiones no se tiene en cuenta a la hora de realizar estudios sincrónicos o diacrónicos a este respecto.

Pero, ¿Cuál es el contexto histórico que nos lleva a la explosión de esta manifestación a caballo entre lo artístico y lo periodístico?

El siglo XX español nace con una de las crisis culturales más profundas que hayamos conocido en el país, que va desde lo cultural hasta lo político y lo estético. Es indudable el rechazo hacia las formas artísticas y los modos heredados del siglo anterior que sufren los intelectuales de la época. Esta negación de lo anterior conlleva un ansía de renovación, de búsqueda de nuevas formas de expresión que se adapten a las necesidades de la cultura occidental, ya agotada del todo en la conciencia del artista de principios de siglo. 
La crisis finisecular, es por tanto el primero de los caminos que ha de conducirnos hacia una nueva forma de expresión hasta entonces desconocida en España y prácticamente inexistente en el resto de Europa.

Muestras evidentes de esta crisis, y ejemplos de renovación cultural y educativa son la Institución Libre de Enseñanza (a la que se encontraba ligado a través de la figura de su tío Eduardo de Ontañón, fundador y director de Parábola, una de las muestras más importantes de este tipo de publicaciones surgida en Burgos en 1923) o el Ateneo de Madrid, centros donde empezaba a fraguarse el espíritu de la revista literaria (con clara idea de grupo de intelectuales burgueses más o menos conocidos que proponen intercambio de ideas y con un lugar común para las tertulias, como veremos más adelante). No podemos olvidar que el equipo que compone cada revista comparte mucho en la empresa que se inicia, y cada uno de los miembros pone una increíble pasión en lo emprendido. La fragua del grupo de intelectuales reunidos en una tertulia es una imagen clarísima de la literatura de vanguardia y de la década de los años 20, que será la que nos ocupe en este estudio. Si pensamos en los casos burgaleses bien conocidas son las reuniones de "El ciprés" y "El Miraflores", aparentemente tascas que dieron lugar a dos grupos de intelectuales bien definidos que se ocuparon de la redacción de las dos publicaciones líderes en la ciudad en cuanto a la revista literaria: la ya citada "Parábola" y la aventura vanguardista liderada por Antonio L. Bouza que abarca la época comprendida entre 1969 y 1985, "Artesa". También es evidente el núcleo de unión que fue la Universidad Popular Segoviana en el caso de la publicación de "Manantial" (1928-1929) y la influencia que la Institución Libre de Enseñanza causó en Gerardo Diego, director de "La cotorra", surgida en 1922 en Soria.

Así, la revista literaria nace con un espíritu de grupo y colectividad que le es inherente y el equipo de estas publicaciones es su alma. No solamente es importante el director, sino que también son clave redactores, saludadores, mentores y firmas invitadas. Lo curioso es que estas revistas aúnan entre sus colaboradores nombres tan conocidos como desconocidos hoy en día, pero sin duda todos gozaban de una tremenda fama en el momento en el que escriben.

\section{La revista literaria en Castilla y León como escaparate}

¿Cómo es posible que muchos de estos autores hayan pasado a formar parte del canon y otros hayan quedado relegados al estudio literario o el anonimato? La experiencia lectora invita a pensar que estas revistas eran utilizadas como plataforma o escaparate para muchos de estos autores, por tanto existe una cierta función publicitaria en los fines de estas empresas, que sin duda, dio lugar a casos exitosos, como será el lugar de las tres revistas que ocupan este estudio. Es tal este afán que en muchos casos nos encontramos con que estas revistas viven de una autofinanciación que hace pensar que si este trabajo no reportaba beneficios económicos algo debería traer de ganancia a los colaboradores: se trataba de un escaparate donde darse a conocer. Esta idea de cantera publicitaria nos lleva a entender los frecuentes intercambios que se daban entre colaboradores de diferentes revistas, y no es extraño encontrar un mismo autor formando en dos o más de ellas. Por poner algunos ejemplos, Gerardo Diego colabora en algún número de "Manantial" y dirige "La cotorra", Machado y Ontañón, firmas habituales de "Manantial" lo son también de "Hora de España" (1937-1938) 
Claro es el caso de "La cotorra" y su sección "Anuncios cotorrescos" en la que podemos ver claramente cómo la publicidad da lugar a buena parte la sustentación económica de la revista, sin perder el carácter jocoso que definía a la revista, como observamos en el siguiente ejemplo:

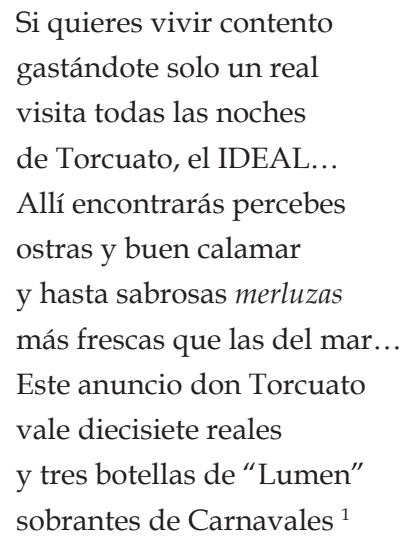

También encontramos láminas publicitarias en "Parábola", que dejan huella de este hecho.

Queda por tanto evidenciado el carácter colectivo y propagandístico de estas publicaciones pero hemos de resaltar aún un rasgo más que define al equipo de estas revistas: el elitismo. Los componentes de estos grupos son siempre intelectuales pertenecientes a la burguesía y con un cierto carácter exclusivista en sus escritos. Las revistas literarias no están escritas para la lectura popular, sino para un público intelectual que encontrará en ellas la vía de reflexión que necesita en ese momento. Cabe en este momento hacer un pequeño apunte referido a "La cotorra", que pueda parecer por su carácter cómico una revista más popular. Cierto es que el público objetivo de la revista es más abierto que en casos como el de "Manantial", con un claro espíritu poético que nos hace pensar en un público más intelectual. Sin embargo, la revista soriana es, en realidad, por su espíritu jocoso de humor inteligente y por muchos otros rasgos (entre ellos la dirección de dos autores bien conocidos dentro del canon de los clásicos españoles) un antecedente claro de una de las revistas más famosas de la cultura española de todos los tiempos: "La codorniz" (1941-1978). Nadie se atrevería a dudar del poco valor intelectual de "la revista más audaz para el lector más inteligente" (lema de la publicación, bien conocido en el mundo cultural de la época), y por tanto lo mismo hemos de pensar del humor matizado de "La cotorra".

Estas publicaciones sirvieron además de cantera para nuevos autores que se dieron a conocer a través de ellas, ya fueran de publicación más espaciada en el tiempo (es decir, con un carácter más claro de revista) o en periodos semanales o quincenales (de forma más cercana al periódico). Tal es el caso, por ejemplo, de Dionisio de la Cruz, que se da a conocer en "Parábola" apadrinado por el joven crítico E. Salazar y Chapela. No es este un dato casual si tenemos en cuenta otra de las características más evidentes de las revistas literarias: su espíritu crítico. Estas publicaciones, en busca de ser un escape

\footnotetext{
1 Díez Revenga (ed.) (2003, nº1, 12 de marzo de 1922).
} 
y una aportación clave para el mundo intelectual, por ello es fácil encontrar en ellas reseñas, recomendaciones y noticias sobre el panorama cultural del momento. Sin duda, los colaboradores de estas revistas compartían un espíritu de mejora que se deja ver en sus textos, ya sea en forma de ensayo, a través de escritos literarios o utilizando el humor como medio de convencimiento.

Muchas son las formas bajo las que se presenta este espíritu crítico, y es precisamente esta otra de las características más propias de la revista literaria: su eclecticismo. Por un lado, podemos encontrar colaboradores de una sola generación o de varias, trabajando hacia un mismo horizonte común (lo que siempre se respira en estas publicaciones es el carácter de grupo bajo la estela del fundador). Además, es indudable la multiplicidad de géneros que aparecen bajo un mismo título. Una revista literaria puede aglutinar ensayos, estudios lingüísticos y literarios e incluso representaciones de diversas artes (pensemos en las láminas de "Artesa" acompañadas de diversos poemas de la época o en los dibujos de "Parábola"). Por tanto, es claro el carácter multivalente de estas revistas.

\section{La revista literaria en Castilla y León como fuente de noticias}

Es fácil pensar, observando las características de estos textos y el contexto del siglo XX, sin el cual sería imposible entender el porqué de estas publicaciones, que sean, como son, un excelente reflejo de la sociedad del momento. La inmediatez de la que gozan estos escritos es tanta como la de un periódico actual, pues se hacen eco de las últimas noticias y novedades en el sector cultural, social y político. Solamente tenemos que observar las noticias de "Parábola", los "cotilleos" que buscan la crítica satírica de "Manantial", el carácter informativo de "Cruz y raya", fundada por Bergamín en 1933; o el duro reflejo de la guerra en "Hora de España", por citar algunos. Así, a pesar del fragmentarismo de la revista literaria - respecto a sí misma y a sus números - la lectura completa de los ejemplares de una de las publicaciones nos llevará a una excelente comprensión de la sociedad y la cultura del periodo de vigencia de la misma.

Reflexionaremos, muy brevemente, sobre tres revistas surgidas en el seno de Castilla durante la década de los años 20, en tres ciudades que comparten el rasgo más provinciano de la ciudad pequeña de Castilla y que pueden servirnos de claro ejemplo en cuanto al espíritu renovador de la región, contrario, en principio a ese carácter provinciano: "La Cotorra" (Soria), “Manatial” (Segovia) y "Parábola” (Burgos).

\section{El caso de "La Cotorra" (Soria)}

En el caso de La Cotorra es imprescindible tener en cuenta a su líder: Gerardo Diego. Harto conocida es la relación de este autor con el mundo de las revistas, pero siempre a través de la revista "Carmen" y de su suplemento "Lola", que comenzaron a publicarse en Gijón en 1927. Sin embargo, menos sabida es la relación previa de Gerardo Diego con el mundo de la revista literaria: la interesante experiencia de edición de "La Cotorra" en 1922.

En abril de 1920 Gerardo Diego toma posesión de la Cátedra de Lengua y Literatura Españolas del Instituto de Soria. Ya entonces entablará amistad con Santiago Gómez Santacruz (futuro redactor de "La Cotorra" en el artículo de salutación del primer número) en la Casa de las Isidras, donde se instaló. 
Según Antonio Gallego Morell, biógrafo del autor, Gerardo Diego no tardó en hacerse con un círculo de amistades entre sus colegas de profesión a pesar de su joven edad. Gerardo Diego se erigió como la representación de una modernidad que empezó a llamar la atención en una sociedad tan cerrada como la soriana de aquella época. Circulaban rumores sobre él, como que deseaba alquilar un piano y subirlo a su habitación, que se convirtieron rápidamente en la comidilla del lugar. Resulta interesante, si tenemos en cuenta que este estudio se centrara en la vanguardia rescatada de las revistas literarias de los años 20 en Castilla y León, que Francisco Javier Díez Revenga lo define en estos momentos del modo siguiente: "enlutado como un seminarista, el joven "ultraísta" encaja en su claustro del Instituto". ${ }^{2}$

El propio Gerardo Diego reflexiona en "Cuatro Glosas", Tercer programa, abril, mayo, junio, 1966 sobre la buena reflexión que tuvo con sus compañeros de Instituto, lejos del ambiente del que Machado le habló trece años antes.

Gallego Morell nos habla en especial de la relación de Gerardo Diego con Tudela (parte fundamental en la redacción de "La Cotorra") y de Granados, que prestó como medio de transporte habitual del grupo su "Forín", un viejo modelo de la marca Ford.

Y el testimonio del poeta da la lista glosada con impresiones personales de la relevancia de cada una de las personalidades de este grupo soriano: "Pero a mí me divertía más charlar y pasear con los bien pronto amigos de la peña del Casino Numancia, varios de ellos coincidentes en aficiones literarias y artísticas. No sería entonces frecuente en una mínima ciudad española - la mínima, por su población, capital de provincia - disfrutar del trato y de la amistad de un arqueólogo de cómo Blas Taracena, un inspector de Magisterio como Gervasio Manrique, de un poeta y abogado como Mariano Granados, de un banquero en ciernes como Epifanio Ridruejo, de un profesor de Normal como Pedro Chico, de un telegrafista poeta como Virgilio Soria, de un conversador con tanto ingenio como Alfredo Llorente, de un odontólogo como Mariano del Olmo. De entre ellos, algunos han muerto ya, no sin dejarnos harto consuelo su memoria. Otros ascendieron a puestos importantes en la vida. Y todos, más o menos, han dado muestras de sus aptitudes para el cultivo de la literatura, ya como aficionados, ya como profesionales. Todos eran, no hay que decirlo, tan jóvenes como yo, pocos años más o menos. Por lo mismo, nuestra tertulia sedente o ambulante era cosa aparte de amistad respetuosa que nos unía a otras personas mayores, como el Abad de la Colegiata, arqueólogo, como el médico don Mariano Î́niguez o como el "buen amigo" de Machado, José María Palacio, con el que tanto hablé yo del poeta de Campos de Castilla. No tardaría en encajarse en nuestra tertulia, siquiera fuera en cortas temporadas de permiso, José Tudela, el mismo Pepe Tudela que iba a servir de motivo para un famoso ensayo de Ortega. Tudela era archivero de Segovia y allí había cuajado otro grupo amistoso presidido por la incomparable bondad e inevitable maestría del Catedrático de Lengua Francesa don Antonio Machado. Tudela nos traía las últimas noticias de don Antonio y los recuerdos de sus antiguos alumnos - varios de los nombrados lo habían sido en Soria, como después un pariente de Epifania, el futuro poeta Dionisio Ridruejo lo iba a ser en Segovia - , y los versos sabidos y recitados de memoria del predilecto poeta eran motivo constante de nuestros coloquios." ${ }^{3}$

De aquí extraemos varios nombres harto conocidos en la redacción de la revista. A esta tertulia se irían añadiendo con el tiempo Bernabé Herrero (futuro cuñado de Tudela) y Ángel Río.

\footnotetext{
2 Díez Revenga (2003: 196).

3 Díez Revenga, Francisco Javier (2002), La revista La Cotorra (Soria, 1922). En la primavera temprana de Gerardo Diego.
} 
De este modo se fragua una comunidad que no es de extrañar, crea una revista, un periódico festivo, de venta los domingos y que se vendía por diez céntimos.

Quien se introduzca de algún modo en la revista, como estudioso o como lector se dará cuenta de las buenas intenciones de la revista. A pesar de su título y del afán jocoso y divertido de la misma no se buscaba ofender a ninguno de los citados en la revista ni de hacer leña del árbol caído, sino de hacer notar lo sucedido en la ciudad, siempre con sumo cuidado de no dañar el ánimo de los convecinos.

\section{El caso de "Manantial" (Segovia)}

En el caso de "Manantial" el contexto histórico cobra vital importancia al tratarse de una revista absolutamente ligada, por los años en los que tiene lugar su publicación (19281929) a una explosión cultural de la vida segoviana.

En este contexto, no podemos olvidar dos hechos clave, que se ligan al año 1919: la llegada de Antonio Machado, que permanecerá hasta 1932, a la ciudad, y la creación de la Universidad Popular Segoviana. La presencia del autor de Campos de Castilla en la ciudad dejará su huella. Es él el responsable de la creación de la Liga Provincial de los Derechos del Hombre en 1922, en la que participaron otras personalidades como Unamuno.

Además, es obligatorio destacar que nos encontramos en un periodo de cambio, que siempre da lugar a nuevas manifestaciones culturales. En un contexto de crisis, revolución y problemas políticos surge una oposición de un grupo de intelectuales burgueses, como demuestra el mitin político celebrado el 14 de febrero de 1031 en el Teatro Juan Bravo, en el que intervienen Ortega y Gasset, Ramón Pérez de Ayala y Gregorio Marañón, entre otros.

En el contexto literario, y dada la naturaleza de este estudio, es importante hacer hincapié en que nos encontramos en el momento de auge de las vanguardias. En 1918 el manifiesto del ultraísmo defiende a ultranza la "necesidad de un arte nuevo" y de lanzar "un grito de renovación". Este será el punto de partida para la generación vanguardista comprendida entre los años 1925 y 1935, que luchará por crear un lazo de unión entre las literaturas española y europeas. En 1925 se publica La deshumanización del arte de Ortega y Gasset, que nos dará las tendencias que debe seguir el nuevo estilo. Este autor, funda además en 1923 "La revista de occidente", publicación clave, tanto si hablamos de vanguardia como si hablamos de revistas literarias. Cerca de esta fecha tienen lugar publicaciones como Sobre los ángeles de Rafael Alberti en 1929 o Poeta en Nueva York de Federico García Lorca en 1929 y 1930. Está claro que algo está cambiando, y en Segovia ese cambio también fue notorio y necesario. Por tanto, nos encontramos con una publicación en la que sí se hará notorio el nuevo arte que se estaba fraguando en España, y en el que la vanguardia se entenderá de otro modo distinto a lo que hemos observado en "La cotorra", pero que tiene en común con la anterior revista el espíritu castellano y el aire de revista de ciudad de provincias, dicho esto en el mejor sentido de la expresión.

\section{El caso de "Parábola" (Burgos)}

Si hemos adscrito el nacimiento de "La Cotorra" a la figura de Gerardo Diego y "Manantial" a la existencia y eclosión de la Universidad Popular Segoviana no podemos dejar de lado que "Parábola" no es sin su creador, líder, director, "poliautor" y mecenas Eduardo de Ontañón. 
La mosca de periodista y escritor de Eduardo le viene por parte paterna. Su progenitor, Jacinto de Ontañón, ya legó sus esfuerzos a publicaciones como "El eco popular", "El independiente" o el conocido "EL Papa-Moscas", que hacía gala de un profundo sentimiento burgalés desde el mismo título (referido al reloj más famoso de la catedral de la ciudad). De su matrimonio con Manuela Levantini, treinta años más joven, nace Eduardo. No conviene olvidar tampoco que José Ontañón, su tío, fue uno de los primeros profesores de la Institución Libre de Enseñanza, lo que abriría puertas en un futuro a Eduardo, que conocería diversas personalidades a través de este vínculo.

Con la muerte de Jacinto en 1917 es Eduardo quien comenzará a ocuparse de "El Papa-Moscas", entendiendo que es el hombre de casa. A pesar de no haber terminado sus estudios de bachillerato al joven se le abren muchas puertas en el ámbito de la burguesía burgalesa, con el recuerdo de su padre como llave. Cabe destacar que la publicación deja huella en Eduardo, por ser la primera en la que tomará parte como director, y por su carácter regionalista, o más bien "defensor de los derechos regionales" (soplo que bien se dejará notar en "Parábola".

Pasaron los años y Ontañón continuó trabajando para lograr sus aspiraciones como poeta y periodista:

Eduardo mantenía muy viva su inclinación periodística nacida a la sombra de "El PapaMoscas". Sus artículos comenzaron a aparecer no sólo en la prensa local -fundamentalmente "Diario de Burgos" y "La voz de Castilla" - sino también en Madrid y el extranjero. De 1920 a 1924 colaboró con el "Diario Español" de la Habana, ayudado por los contactos que su padre había mantenido en la colonía burgalesa allí asentada. Por aquel entonces, de 1923 a 1925, datan sus artículos en "La Libertad" de Madrid.

Periodísticamente, nuestro protagonista desarrollará un formato a medio camino entre la crónica y el reportaje, que le servirá para ir dando cuenta del mundo que late a su alrededor. Su estilo quiere ser una nueva visión de la realidad, un "re-descubrimiento" de mirar sorpresivo del tradicionalismo que lo rodea. ${ }^{4}$

Este interesante apunte que nos ofrece Fernández de Mata indica ya un hálito del vanguardismo segundo al que se refiere este estudio. Esa nueva manera de ver lo tradicional, no solamente refiriéndose a la renovación en el sentido estético, sino yendo más allá.

En 1923 surge la aventura, en principio periodística, literaria y editorial, de "Parábola". Esta primera andadura de la revista dará lugar a los números de junio y julio de ese año, adscritos al movimiento vanguardista, concretamente al ultraísmo. Colaborarán en ellos las viejas amistades de "El Papa-Moscas", como Saiz Barrón y Arasti, otros nuevos conformantes del núcleo burgalés como Viedma y autores bien conocidos como Fernando de la Quadra Salcedo, Rafael López de Haro, Amado Caballero y González de Ruano.

Repasando el equipo de redacción daremos unos pequeños apuntes sobre los pseudónimos utilizados por el propio Ontañón a lo largo de la publicación. Aunque el uso de diversos nombres es hoy para el investigador un juego de despistes donde bien se reconoce el sello del autor en cada uno de los textos, parece ser que el director de "Parábola" lo utilizó como un anzuelo editorial que daba a entender que en la revista colaboraban más autores de los que en realidad lo hacían. Sin embargo, si estamos atentos todas las firmas que se apropia Ontañón son juegos de palabras con su nombre, y muy a menudo con el de su padre: Haddita, Enríquez de Mendoza (uno de los más utilizados por él, que hace juego con el apellido compuesto de su padre), Menéndez o M. Méndez, así como el

\footnotetext{
4 Fernández De Mata, Ignacio - Estébanez Gil, Juan Carlos (ed.) (2004), Parábola (Burgos 1923-1928).
} 
uso de siglas como EE.MM con algunos ejemplos. Hoy, sin embargo, es difícil desligar el uso de estos pseudónimos de un carácter vanguardista. La vanguardia es al fin y al cabo juego, despiste, escondite, transformar la realidad en diversión a través de confusión.

En 1927 la revista comienza una nueva andadura, esta vez más lejana a teorías estéticas cercanas a la vanguardia, pero sí con un espíritu renovador de la visión de Castilla.

“Castilla está en el centro de los objetivos. El subtítulo de la revista así lo confirma: "Cuadernos mensuales de valoración castellana". "Parábola", pequeña nave, barco sin mar, un poco marinero en tierra, desea convertirse en la voz que anuncie el incipiente dinamismo de esta tierra. "Parábola", pretende, en fin, romper el pretendido y ancestral silencio castellano:

"Castilla ha estado en blanco hasta hora en el mapa internacional de las nuevas actividades artísticas; he aquí hoy, buen día de noviembre de 1927, la cordial caja de pinturas que abrimos con la emoción de un niño contento.

Queda pues por tanto evidenciado el carácter vanguardista de la revista en su segunda etapa, entendiendo la vanguardia como una renovación y un punto de progreso en Castilla. En este sentido, debemos destacar que en muchas ocasiones es través de la idea del mar (en esos momentos no ajeno a Castilla). El propio Ontañón utiliza en diversas ocasiones imágenes referentes a lo marítimo en sus poemas y en sus textos.

En esta etapa la revista cuenta con el apoyo que le presta María Teresa León desde su exilio argentino, que dirige la revista "Burgos", revista oficial del centro burgalés en el país. En el número correspondiente a julio de 1928, que a pesar de no estar firmado por la autora, es de autoría fácilmente reconocible hace mención a "Parábola" e indica sus posibilidades para hacer renacer Castilla.

Volviendo al equipo de redacción hay que destacar una figura clave: la del ilustrador, que creará una imagen y un estilo característicos para la revista. "Parábola" no es sin Jaime Prada. Casualmente, el ilustrador fijó su residencia en Burgos desde 1922 hasta 1930, años en los que se encuentra en contacto directo con Ontañón y en los que "Parábola" nace, se desarrolla y muere. Además, colabora con el director de la revista en dos textos anteriores al nacimiento del proyecto periodístico y de corte mucho más tradicional: Breviario sentimental y Sinfonía azul (este último precisamente se lo dedica Ontañón al artista).

Como ocurría en el caso de "Manatial", el fundador y director de "La Cotorra", Gerardo Diego, colabora también con "Parábola en el año 1927 con "Tres Canciones".

Del mismo modo, otros colaboradores de "Manatial" prestan su pluma a la publicación, como es el caso de Alfredo Marquerie, en el año 1923 con "Paisaje Urbano".

Así, se configura la lista de colaboradores de la publicación: José María Alfaro Polanco, Fernando Allué y Morer, Marcelino Álvarez Cerón, Lluis Amado Caballero, Antonio José, Eduardo Arasti Arrizabalaga, César M. Arconada, Francisco Ayala y Garcái Duarte, Augusto María Casas, Juan Chabás Martí, Leopoldo Cortejoso Villanueva, Francisco y José María de Cossío, Juan Díaz Caneja, Juan Manuel Díaz Caneja, José Díaz-Fernández, Gerardo Diego, Roberto Escribano Ortega, Ángel Espinosa, Rafael Ferrer Fornes, Federico García Lorca, José de Gadorqui y Uirdanabia, Ernesto Giménez Caballero, César González Ruano, Benjamín Jarnés, Juan Lacomba, Rafael López de Haro, Ernesto López-Parra, López Prudencio, Maruja Mallo, Alfredo Marqueríe Monpín, Rafael Marquina, Francisco Martín y Gómezm Francisco Mateo, Concha Méndez, Manuel Méndez Domínguez, Eduardo de Ontañón, Santiago Ontañón, Teófilo Ortega, Julián María Otero 
Rubial, Miguel Pérez Ferrero, Jaime Prada Losada, Fernando de la Quadra Salcedo, Luis Sáiz Barrón, Pedro Salinas, Francisco Santa Cruz, José María Vela de la Huerta, Mario Verdaguer, Juan Vidal Martínez, Alfonso de Viedma y Francisco Vighi Fernández.

Nótese que nombres de la talla de García Lorca o Pedro Salinas hubiesen sido imposibles de no ser por el acceso a la Institución Libre de Enseñanza que le brinda a Ontañón su tío José.

Así, queda configurado un equipo de redacción que me atrevería decir que no es otro que el propio Ontañón (bajo una serie de pseudónimos) y el ilustrador Jaime Prada, configurándose la revista como un haz de colaboraciones provenientes de antiguas amistades procedentes de "El Papa-Moscas", nuevos conocidos a través de contacto en Madrid y figuras de la cultura de Castilla y del panorama literario y periodístico español. Este equipo dará lugar a dos etapas de vanguardia de la revista: la primera con un ultraísmo muy cercano a los poemas de "Manantial" y la segunda marcada por un claro espíritu de renovación de Castilla.

\section{Conclusiones}

Como hemos observado, la revista literaria, cuyo auge se establece en el siglo XX en España tuvo grandes frutos en Castilla, como demuestran las tres publicaciones a las que hemos hecho breve mención en este estudio y otras muchas como "Claraboya" (León), "Meseta" (Valladolid) o "El cobaya" (Ávila).

Este tipo de publicaciones da una muestra clara de la conciencia de vanguardia, entendida como cambio, renovación, lucha, progreso y es el escaparate de la nueva literatura de vanguardia que llegó a instaurarse como tal en España con mucha menos fuerza que en otros países de Europa, pero que sí dio algunos coletazos dignos de mención en nuestro país. Tradicionalmente la vanguardia se ha adscrito a otras regiones de España, pero si bien es cierto que quizá este tipo de manifestación artística tuvo grandes frutos en otros lugares del país, Castilla no se quedó corta en el progreso, la innovación y la experimentación literaria. Lo que se ha pretendido es dar un aire fresco a la idea de Castilla como un territorio seco, árido, duro y de gentes ajenas al progreso que le ha sido concedido tradicionalmente.

La revista literaria, como vehículo de propaganda y autopromoción, como publicación de carácter ecléctico y colectivo, dio frutos inesperados e irreverentes en cierto modo, pero que dan muestra de la realidad de un momento concreto. En este sentido, hemos encontrados firmas harto conocidas hoy día y que el canon ha decidió incorporar como clásicos de la literatura española y otras que pasaron a la historia con la misma fugacidad que estas publicaciones, casi siempre sostenidas económicamente por el equipo redactor, que creyó, con Gerardo Diego, Machado y Ontañón a la cabeza, que la empresa que sostenían era digna de ver la luz. Lo que es innegable es que la lectura de las tres publicaciones, nos dan el espíritu y el aire de una Castilla en los años veinte donde se estaban fraguando nuevos modelos literarios y donde una nueva burguesía intelectual quería cambiar.

"La Cotorra" supone un cambio en la manera de contar, en la forma de hacer periodismo y literatura de forma seria, pero a través del juego. $Y$ ese es el punto en el que debemos entender que la publicación es vanguardista.

"Manantial" por su parte, se abrió ya al arte gráfico con las láminas de Lozoya y otros dibujos de José Machado e incluso fotografías de esculturas de Emiliano Barral. Supo 
Las revistas literarias en Castilla y León en los años 20. Un fenómeno a la cabeza de la vanguardia

dar muestra de una poesía casi vanguardista, pero que no dio los pasos suficientes como para poder ser considerada ultraísta y ante todo, abrió la ventana de Castilla al cosmopolitismo del que se hacía gala en Antena de Manantial.

"Parábola" supo fraguar, a través de su líder, Eduardo de Ontañón, un nuevo modelo de Castilla, defendiendo su renovación y abriendo la lista de firmas a personajes provenientes de su relación con la Institución Libre de Enseñanza en su segunda etapa y haciendo gala del ultraísmo más puro que podía darse en un momento como ese y en una ciudad como Burgos en sus primeros números.

Tres revistas, tres ciudades, tres líderes y tres formas de entender la vanguardia que comparten el provincianismo por un lado, y el ansia de renovación por otro y que dan muestra de una sociedad castellana en los años veinte en la que una serie de intelectuales intercambian firmas en publicaciones de este tipo, comparten ideas y creen, tal y como nos dan a entender sus páginas, en el cambio y la renovación, a través de diversos caminos.

\section{Bibliografía}

Albadalejo, Tomás et al. (ed.) (1992), La vanguardia, renovación de los lenguajes poéticos, Madrid: Júcar.

Alonso, Cecilio (1985), «Una carta abierta de Antonio Machado a Miguel de Unamuno (1903)», Ínsula: revista de letras y ciencias humanas, Madrid: Ínsula, Librería, Ediciones y Publicaciones.

Alonso, Dámaso (1990), Poetas españoles contemporáneos, Madrid: Gredos.

Blanch, Antonio (1976), La poesía pura española, Madrid: Gredos.

Bonet, Juan Manuel (1995), Diccionario de las vanguardias en España, 1907-1936, Madrid: Alizanza.

Bousoño, Carlos (1966), Teoría de la expresión poética, Madrid: Gredos.

BrinUEGA, Jaime (1981), Las vanguardias artísticas en España, 1909-1936, Madrid: Istmo.

BuCKLEY, Ramón - CRISPÍN, Juan (eds.) (1973), Las vanguardias españolas (1925-1935), Madrid: Alianza.

Burguera NAdAl, María Luisa - Fortuño Llorens, Santiago (eds) (1998), Vanguardia y humorismo. La otra generación del 27, Castellón: Universitá Jaume I.

Burger, Peter (1987), Teoría de la vanguardia, Barcelona: Península.

CANo Ballesta, Juan (1972), La poesía española entre pureza y revolución, Madrid: Gredos.

Cernuda, Luis (1970), Estudios sobre poesía española contemporánea, Madrid: Guadarrama.

Delgado, Agustín (et al.) (1971), Equipo "Claraboya": Teoría y temas, Barcelona: Saturno.

Díez De Revenga, Francisco Javier (ed.) (2003), La cotorra (Soria, 1922), Burgos: Fundación Instituto Castellano y Leonés de la Lengua.

Díez De Revenga, Francisco Javier (1998-99), «La Revista La Cotorra (Soria,1922) en la primavera temprana de Gerardo Diego», Revista de la Facultad de Filología, Tomo 48-49, 1998-1999, 195-218.

Domínguez Rey, Antonio (1983), «En torno a una antología poética de criterio múltiple», Nueva estafeta 50, 1983, 61-66. 
Fernández De Mata, Ignacio - Estébanez Gil, Juan Carlos (2004), Parábola (Edición facsímil), Burgos: Fundación Instituto Castellano y Leonés de la Lengua.

GEIST, Anthony Leo (1980), La poética de la generación del 27 y las revistas literarias: de la vanguardia al compromiso (1818-1936), Madrid: Guadarrama.

HernANDO, Miguel Ángel (1975), Prosa vanguardista en la generación del 27 (Gece y "La gaceta literaria"), Madrid: Prensa Española.

Lanz, Juan José, La revista "Claraboya" (1963-1968: un episodio fundamental en la renovación poética de los años sesenta (2005), Madrid: Universidad Nacional de Educación a Distancia.

LECHNER, Joahennes (1968), El compromiso en la poesía española (1898-1936), Leiden: Universite Pers Leiden.

Morelli, Gabriele (1991), Treinta años de vanguardia española, Sevilla: El carro de la nieve.

OsunA, Rafael (2004), Las Revistas literarias: un estudio introductorio, Cádiz: Universidad de Cádiz.

Pérez Barredo, R. (13 de junio de 2004), «Universal Vanguardia Castellana», Diario de Burgos (No 35.694), 24 y 25.

Puerto, José Luis (ed.) (2003), Manantial: (Segovia, 1928-1929), Burgos: Fundación Instituto Castellano y Leonés de la Lengua.

Ramos Ortega, Manuel José (2001), Las revista literarias en España entre "La edad de Plata" y el medio siglo: una aproximación histórica, Madrid: Ediciones de la Torre.

TORRE, Guillermo (1971), Historia de las literaturas de vanguardia, Madrid: Guadarrama.

TORRE, Guillermo (1925), Literaturas europeas de vanguardia, Madrid: Caro Raggio.

VALCÁRCEL, Eva (2000), La vanguardia en las revistas literarias, La Coruña: Universidad de la Coruña.

Videla, Gloria (1971), El ultraísmo. Estudios sobre movimientos poéticos de vanguardia en España, Madrid: Gredos.

\author{
Mercedes Andrés López \\ Fundación Instituto Castellano y Leonés de la \\ Lengua \\ y Universidad de Burgos \\ Palacio de la Isla \\ Paseo de la Isla 1 \\ 09003 Burgos \\ España \\ merandlop@gmail.com
}

